\title{
Sous Vide Cooked Technology and food safety control of chicken
}

\author{
Hui Ding ${ }^{1, *}$ \\ ${ }^{1}$ College of Food Science and Technology, Wuhan Business University, Wuhan, China
}

\begin{abstract}
With the rapid development of food science and cooking technology, people's demand for the quality of food is gradually increasing. A delicious, safe and healthy diet has become a new goal for people. The emergence and the continuous improvement of Sous Vide Cooked Technology technology helps in catering these needs, which has been attarting the attention from more and more consumers. Chicken is rich in nutrition, high in protein and low in fat. Sous Vide Cooked Technology used in chicken cooking preserves chicken's original flavor and color, which also maximumly keeps the nutrients of chicken.
\end{abstract}

\section{Origin of Sous Vide Cooked Technology}

The modern cooking concept is based on the principles of low sugar, low fat and low salt. Chicken is an economical cooking raw material. It is found that every $100 \mathrm{~g}$ chicken contains $167 \mathrm{kcal}, 19.3 \mathrm{~g}$ protein, $9.4 \mathrm{~g}$ fat, $1.3 \mathrm{~g}$ carbohydrate, $69 \mathrm{~g}$ water, $48 \mathrm{ug}$ vitamin $\mathrm{A}, 50 \mathrm{ug}$ VB1, 90ug VB2, 5.6mg VE $0.67 \mathrm{mg}$, 9mg calcium, $156 \mathrm{mg}$ phosphorus, $1.09 \mathrm{mg}$ iron, $251 \mathrm{mg}$ potassium, $63.3 \mathrm{mg}$ sodium, $19 \mathrm{mg}$ magnesium, $11.75 \mathrm{ug}$ selenium, $62-187 \mathrm{mg}$ cholesterol, and many other mineral substance and vitamins [1]. Since less fat is present in chicken, if it is cooked in the traditional way, it would be easy for the chicken to be dry and lack of gravy. Nevertheless, if Sous Vide Cooked Technology is applied, cooked chicken will readily be tender and juicy, keeping the chicken breast to the maximum extent of moisture and nutrition.

The procedure of Sous Vide Cooked Technology (Sous-Vide) involves vacuum packing the food, then putting the entire bag into hot water that has been adjusted to the desired temperature and allowing it to cook slowly for hours or even days.

Sous-Vide was introduced by the French chef George Pralus in 1970s, which was one of the greatest kitchen revolutions. It brought about great changes in the commercial cooking scene over the past few centuries. This approach differs from traditional slow cooking in that sous vide cooking requires the ingredients to be vacuum-packed and then placed in a water bath at a temperature below $100{ }^{\circ} \mathrm{C}$ (usually $50^{\circ} \mathrm{C}$ to $90^{\circ} \mathrm{C}$ ). Sous-vide cooking was pioneered in the 1970s and perfected by food makers in 2000 .

\section{Principle of Sous Vide Cooked Technology}

Sous Vide Cooked Technology is called "accurate scientific cooking", which utilizes cryogenic cooking machine. This cooking method needs two elements to support: control of constant temperature and precise time. The key point is to allow the ingredients to be cooked accurately to the point where they are just ripe, when the cooking effect is perfect. Otherwise, if the time and temperature are not accurately set, the cooked food will be raw or overcooked and affect the taste. For example: meat raw materials after processing and vacuum treatment, the temperature is set at around 60 degrees, where air out of contact with the meat during the process of cooking. After long time of boiling, meat fibres are destroyed, making the meat soft and gelatinous.

Sous Vide Cooked Technology triggers chemical reactions (protein denaturation, hydrolysis) of compounds in food. Because there is no oxygen in vacuum packaging in cooking, there is no oxidation reaction in the cooking process, which means that even subtle flavours are thus kept stable. There is no gradient change in the degree of meat cooked in this way, while the part outside the water tub will be overcooked. If food is cooed in the traditional way, $15-20 \%$ of the food weight is lost, most of which is the moisture in the food, making the food overdone. According to a previous study [2], when food is cooked with vacuum and low temperature, the water loss is only between $5 \%$ and $8 \%$, and the food will be particularly fresh and tender, which also maximizes the original flavour and colour of the food. It reduces the use of salt, retain the nutrients of food, is better in preserving the vitamins in the food than steaming, and requires little amount or is free of cooking oil. Therefore, Sous Vide Cooked Technology is suitable for human absorption.

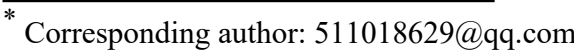




\section{Influencing factors of Sous Vide Cooked Technology}

\subsection{Effect of curing degree on Sous Vide Cooked Technology Chicken.}

After the initial processing of raw materials, the taste and time of pickling directly determine the taste and flavour of chicken. The pickling method is to add salt, white pepper and western vanilla to the boneless chicken for seasoning and pickling. If the pickling time is too short, the flavour will be insufficient and the seasoning will not be able to infiltrate into the chicken tissue. If the pickling time is too long, the chicken will lose water and taste too salty, which affects the food quality. In an experiment where chicken was pickled with 15 and 35 minutes respectively, it was found that the taste of chicken pickled for 15 minutes is a little light, where the scent of spices stays mostly on the surface of chicken, whereas chicken pickled for 35 minutes had relatively better taste and was more acceptable.

\subsection{Effect of vacuum Packaging on Sous Vide Cooked Technology Chicken.}

The main advantage of vacuum packaging is that the heat is efficiently transferred to the ingredients by water bath, rather than through the air in the heating bag. Vacuum packaging can eliminate the oxygen around the food, and thus reduce the possibility of oxidation during storage and cooking. It helps to reduce the peculiar smell and discoloration of food, and prevent food from external pollution in the process of transportation. Vacuum packaging is divided into three degrees: low, medium and high. The vacuum degree is to realize three different vacuum extraction states by adjusting the vacuum time and pressure of the vacuum compressor. Low vacuum is not suitable for low temperature cooking, medium vacuum is mainly used for low temperature cooking of meat and poultry, and high vacuum is for cooking vegetables and fruits, such as carrots, onions, and various fruits.

In the production of chicken cooked in Sous Vide, medium vacuum packaging should be strictly adopted. If there is air in the bag, the sample will float on the water surface during cooking, which will lead to entering of water into the bag, loss of flavour substances, and the inability to ensure food hygiene and safety. As a result, tase of the food is affected.

\subsection{Effect of temperature setting on Sous Vide Cooked Technology Chicken.}

The temperature of Sous Vide Cooked Technology set for chicken is the temperature that the centre of meat needs to achieve. After cooking, the internal and external temperature needs to be the same, which can maximize the freshness, moisture, taste, delicacy and nutrition of chicken raw materials. If temperature for Sous Vide Cooked Technology is set too low, cooking time will be prolonged and chicken is not cooked. If the temperature is too high, chicken will be overdone, making the taste tough and the texture rough. In practice, $1.5 \mathrm{~cm}$ thick chicken leg can achieve the best taste when cooked at $66^{\circ} \mathrm{C}$. Sous Vide Cooked Technology, using accurate scientific heating temperature and time, not only achieves the best treatment of chicken, but also retains most of the nutrients in the food, making the chicken taste better, delicious, less moisture loss, soft and tender. Precise temperature control makes the cooking process easy to successfully copy and more readily to grasp the raw and cooked degree of the food.

\subsection{Effect of heating time on Sous Vide Cooked Technology Chicken.}

The time for Sous Vide Cooked Technology is determined by the thickness of the chicken, which refers to the time it takes for heat to infiltrate into the chicken centre. Compared with thinner chicken raw materials, thicker chicken raw materials take longer time at the same low temperature. In low-temperature cooking, a thermometer is inserted into the centre of the chicken raw material, and when the central temperature reaches the set temperature, the time for the chicken to reach the set central temperature can be calculated. Time set for Sous Vide Cooked Technology also affects the quality of chicken, too short cooking time causes chicken undercooked, making the proteins not completely deteriorated, whereas too long cooking time leads to chicken aging, hardening, and loss of taste. In practice, it is found that the texture of chicken can reach the best condition when using $1.5 \mathrm{~cm}$ thick chicken leg at $66{ }^{\circ} \mathrm{C}$ and heating for 1 hour. The heating temperature and time for common ingredients are summarized in Table 1 [3].

Table. 1. Summary of Sous Vide cooking temperature and time for common ingredients.

\begin{tabular}{|c|c|c|c|}
\hline $\begin{array}{c}\text { Raw } \\
\text { material }\end{array}$ & $\begin{array}{c}\text { Heating } \\
\text { temperature }\end{array}$ & $\begin{array}{c}\text { Heating } \\
\text { time }\end{array}$ & Remarks \\
\hline Beef steak & 59 & $60-120$ & $2.5 \mathrm{~cm}$ Thickness \\
\hline Lamb & 61 & 90 & $3.8 \mathrm{~cm}$ thick \\
\hline $\begin{array}{c}\text { Streaky } \\
\text { pork }\end{array}$ & 82 & $24-48$ & The streaky \\
\hline Chicken & $64-67$ & 60 & $\begin{array}{c}\text { Heat the whole } \\
\text { bone-in chicken } \\
\text { leg for extra } 30 \\
\text { minutes }\end{array}$ \\
\hline Cod & 60 & 20 & $2.5 \mathrm{~cm}$ thick \\
\hline
\end{tabular}

\subsection{Advantages of Sous Vide Cooked Technology over tranditional cooking method.}

In traditional cooking method of boiling, food loses some of its nutrients, such as water-soluble vitamins in the process of boiling. For traditional cooking, the most nutritious way to eat is to drink with soup. Compared with the traditional cooking method, Sous Vide Cooked Technology has the following advantages:

1) Keep the original flavor and aroma of the food as much as possible.

2) Retain the color of food.

3) Reduce or eliminate the use of salt. 
4) Retain the nutrients of the food and separate the raw juice and water of the food.

5) Retain vitamins better than traditional steaming and cooking.

6) Does not need or use a small amount of cooking oil.

7) Ensure that the quality standards are consistent after each cooking.

\subsection{Advantages of Sous Vide Cooked Technology operation.}

1) The weight of the finished product can be accurately controlled by minimizing the weight loss.

2 ) It can greatly improve the taste of ordinary meat.

3) The rest can be refrigerated to minimize waste.

4) Compared with gas stoves and ovens, it can save energy.

5) It can reduce the oil fume pollution in the kitchen.

6) Different food can be cooked at the same time through separate packaging.

7) Time and temperature are easy to control, which achieve the desired effect.

8) It has more preparation time: Sous Vide Cooked Technology can maximize the preparation of the kitchen in advance, and the food cooked in vacuum can be frozen or refrigerated and heated again when needed [4].

\section{Food safety control.}

When cooking food, people are used to frying, deepfrying, stir-frying and charcoal barbecue at high temperature for a long time, thinking that the color, smell and taste of the food cooked in this way will be better.

\subsection{Reduce the risk of cross-contamination of food during storage.}

Food is cooked after being sealed in vacuum, which can quickly heat the food through the heat transmission by water, reducing the risk of cross-contamination during storage, thus prolonging the shelf life of the food. At the same time, Sous Vide Cooked Technology can accurately and scientifically produce the quality that cannot be achieved by traditional cooking methods, such as restraining the peculiar smell produced in the process of food oxidation, reducing the evaporation loss of water and flavor volatiles during cooking, and preserving the original flavor of food [5].

Temperature set for Sous-Vide, in principle, should be equal to or greater than 65 degrees for sterilization. 4 to 65 degrees is the ideal temperature for bacteria growth in food. The moisture loss of food can be reduced by controlling it within 70 degrees in Sous Vide Cooked Technology cooking. But the temperature and time required by different food are different. Citing the temperature and time comparison table given by Thomas Keller, a master of molecular cuisine, his dishes have passed the inspection of the United States Food and Health Administration and should be safe.

\subsection{Prevent the potential danger caused by high temperature cooking.}

High-temperature cooking methods such as baking, charcoal barbecue and high oil temperature can produce good taste and attractive scent in the cooking process, such as the crisp texture of American fried chicken and English fish and chips, the crispness of French fries and American onion rings, Turkish mutton kebabs and smoked bacon and so on. In the process of hightemperature cooking, there will be hidden dangers of food safety, in addition to causing nutritional losses, such as vitamins. At the same time, toxic and harmful substances are produced at high temperatures, such as carbohydrates, fats and proteins. Under the condition of high temperature cooking, fatty substances may change, resulting in high temperature oxidation and polymerization of toxicants, as well as polycyclic aromatic hydrocarbons (PAHs) carcinogens. In meat products, under cooking conditions such as charcoal fire barbecue and high temperature smoking and barbecue, saturated fat will produce a variety of carcinogens, including powerful benzo [a] pyrene carcinogens. When cooked with charcoal fire at high temperature, the carcinogens produced will be serious. Such as Turkish roast mutton, Brazilian roast beef, charcoal roast New Orleans chicken wings and other foods, there is also a problem of benzo [a] pyrene pollution in the cooking process. A large number of carcinogens are produced in high-temperature smoked meat when the temperature is higher than $300{ }^{\circ} \mathrm{C}$, which is a significant cause of gastric cancer. Recent research has found that starch foods produce higher concentrations of acrylamides after high-temperature fried cooking, which have been proved to be carcinogenic [6]. Bread, cookies, biscuits and other baked food also contain a small amount of acrylamide on the outside. In the cooking process, the higher the heating temperature, the greater the production of arylamide. In the cooking method of frying, when the temperature exceeds $200{ }^{\circ} \mathrm{C}$, its production will increase rapidly, resulting in the largest number of carcinogens. In daily life, more consideration should be given to the nutrition and health of food and the potential dangers of high-temperature cooking. When making Korean barbecue and Xinjiang mutton kebabs, electric oven is more suitable to bake. In the cooking process of meat, fish and egg products, microwave cooking can also reduce the content of heterocyclic amines. Cooking methods such as steaming, boiling, stewing, burning, and using pressure cookers between $100{ }^{\circ} \mathrm{C}$ and $120{ }^{\circ} \mathrm{C}$ will not produce these harmful substances. Therefore, SousVide cooking is beneficial to human health.

\subsection{Avoid hidden worries about microbial safety of food cooked in Sous Vide Cooked Technology.}

Sous Vide Cooked Technology in the absence of oxygen is not conducive to food spoilage for bacterial growth. If food is stored in a temperature range suitable for bacterial growth for too long, such as a temperature range of 4 to 60 degrees Celsius, it is easy to breed 
anaerobes, such as Clostridium perfringens. The spores of Clostridium perfringens can survive in the cooking process because of their heat resistance. If the temperature is not controlled properly, Clostridium perfringens will germinate and multiply in vacuumpacked food. If food contaminated by Clostridium perfringens is consumed, individuals may suffer from acute gastroenteritis. If the food cooked in Sous Vide Cooked Technology is stored in the refrigerator for a long time, the anoxic and refrigerated environment is conducive to the reproduction of facultative anaerobes such as Botox and Listeria monocytogenes. Failure to cook the food thoroughly in the cooking process of the fish is not enough to eliminate the pathogenic bacteria or parasites in the food. Because of the hidden food safety hazard, all these need to be avoided in the cooking process.

\section{Summary}

Human beings should return to the original and advocate the most natural lifestyle. Low-temperature diet has become popular in European countries. From a cooking point of view, we should choose a low-temperature way as far as possible, which protects the nutritional composition of meat and gives better flavour. It is more representative of our pursuit of authentic taste and leads the health.

\section{Acknowledgement}

This study is funded by construction of Wuhan traditional Food industrialization Engineering Technology Research Centre (Wuhan Science and Technology Plan Project No: 2015021705011608).

\section{Reference}

1. Y. Zhang, B. Hu, Q. Zhang, J. Zhongkai Coll. Agri. and Engi. 22, 66-71 (2009)

2. Q. Lin. Env. 5, 77-79 (2014)

3. Z. Xiao, China Food Safety Mag. 200, 149+151 (2018)

4. T. Wang, China's New Tech and New products. 16, 67-68 (2016)

5. Anonymous, Tibetan Sci. Tech. 2, 63-64 (2005)

6. D. Zuo, AOCQ. 7, 316 (2013) 